\title{
Translational diffusion of flexible lipid chains in a Langmuir monolayer: A dynamic Monte Carlo study
}

\author{
T. Sintes, ${ }^{*}$ A. Baumgaertner, and Y. K. Levine ${ }^{\dagger}$ \\ Forum Modelierung, Forschungszentrum Jülich, 52425 Jülich, Germany \\ (Received 28 September 1998; revised manuscript received 18 March 1999)
}

\begin{abstract}
We report a computer simulation study of the lateral diffusion of conformationally disordered lipid molecules in a monolayer structure. The simulations were carried out with dynamic Monte Carlo methods, employing two different representations of the internal motions of the lipid chains. The classical Cohen-Turnbull theory is found to provide a good description of the simulated lateral diffusion coefficients at moderate densities. The substantial deviations found at low densities are attributed to the small density fluctuations needed to create the free volume required for the lateral diffusion process. [S1063-651X(99)01007-7]
\end{abstract}

PACS number(s): 87.10. $+\mathrm{e}$

\section{INTRODUCTION}

Lateral diffusion of lipids and proteins in cell membranes has been the subject of numerous theoretical and experimental studies. This process has been invoked in the description of many cellular processes [1,2], from directed transport in cellular locomotion [3] to reaction kinetics [4-6]. Lateral diffusion is also considered to play an important role in the determination of submicroscopic domain structures in membranes [7]. Most experimental studies have employed the techniques of fluorescence recovery after photobleaching (FRAP) [8] or pulsed field gradient NMR $[9,10]$ for the measurement of lateral diffusion coefficients. The diffusion constant obtained in this way reflects an average over a large number of molecules and is generally analyzed using the theory of Brownian motion. In particular, the free volume model introduced by Cohen and Turnbull (CT) [11] is used for the extraction of quantitative information.

Deviations from the classical CT theory had, however, been observed in earlier studies of lateral diffusion in lipid monolayers, particularly at high areas per molecule [12]. A description of the dependence of the observed diffusion constant over the entire range of areas per molecules, however, is provided by a fractal treatment [13] that predicts fractal scaling oscillations of the average lateral diffusion.

In order to gain more insight into the physical processes underlying the lateral diffusion in lipid membranes, we have here undertaken a study of the phenomenon in a monolayer assembly using dynamic Monte Carlo techniques. Toward this end, two approaches were used. One utilizes a lattice representation of the lipid chains, and the second is based on an off-lattice approach in which the lipid chains are represented by a string of beads. The lateral diffusion was monitored as a function of the surface density of the monolayer, expressed in terms of the effective area per molecule. Con-

\footnotetext{
*Present address: Instituto Mediterrano de Estudios Avanzados, IMEDEA (CSIC UIB), E-07071 Palma de Mallorca, Spain.

${ }^{\dagger}$ Present address: Section for Computational Biophysics, Debye Institute, Buys Ballo Laboratory, P.O. Box 80.000, 3508 TA, Utrecht, The Netherlands.
}

finement effects were observed in both approaches only at very high densities. The dependence of the simulated lateral diffusion coefficient followed the CT theory at intermediate densities, but large deviations were found at low densities, corresponding to high areas per molecule. These findings are in excellent agreement with the experimental study of Peters and Beck [12]. Interestingly, both models show that the density fluctuations follow the expected thermodynamic behavior across the density range under study. The results indicate that the deviations from the CT theory are simply due to the fact that at low densities, only small amplitude density fluctuations are needed to create sufficient free volume to induce the lateral diffusion of the lipid molecules.

\section{METHOD OF SIMULATION}

\section{A. Knight's gambit moves model}

This lattice technique for simulating the dynamic behavior of hydrocarbon chains of lipids in monolayers and monolayer structures has been discussed and validated in detail previously [14-19] and only the salient points will be summarized here. Essentially, the method makes use of a representation of hydrocarbon chains on a cubic lattice with C-C bond vectors chosen from among the 24 vectors of the set $\{0, \pm 1, \pm 2\}$. The bonds are of length $\sqrt{5}$, so that each lattice unit corresponds to a distance of $0.69 \AA$. The bond vectors used to construct the model chains are chosen from the basis set under the restriction that the distances $R_{12}$ between atom $i$ and atom $i+2$ lie in the interval $\sqrt{10} \leqslant R_{12} \leqslant \sqrt{18}$. The torsional potential $U(\Phi)$ for the rotation about each C-C bond is parametrized by symmetric square wells corresponding to the trans $\left(-30^{\circ} \leqslant \Phi \leqslant 30^{\circ}\right)$ and two gauche states $g^{+}$and $g^{-}\left(155^{\circ} \leqslant|\Phi| \leqslant 90^{\circ}\right)$. The heights of the wells and the intervening barriers were chosen so as to reproduce the values of $\langle\cos \Phi\rangle$ obtained from the continuous potential [20] over the temperature range $200-800 \mathrm{~K}$.

The monolayer consisted of 1000 model lipid chains each 16 atoms long and was contained in a Monte Carlo box having a square cross section in the $x-y$ plane of the lattice and extending along the positive $z$ axis. Periodic boundary conditions were applied in the $x$ and $y$ directions. The head groups of the model chains were attached to the interface at 
$z=0$, by applying a potential of the form

$$
U_{h g}(z)=\left\{\begin{array}{cc}
0 & \text { for } z<0, \\
\frac{1}{2} k z^{2} & \text { for } z>0 .
\end{array}\right.
$$

Moreover, each methylene group was subjected to a repulsive harmonic potential, $U_{c h}(z)$, on crossing the interface where $z<0$. The simulations reported below were carried out on taking $k=1.0 k_{B} \mathrm{~T}$, which mimicked the diffuse nature of the interface.

Only hard-core interatomic interactions were included, so that the total energy of a model chain $U$ is

$$
U=U(\Phi)+U_{h g}(z)+U_{c h}(z) .
$$

The excluded volume effects were implemented by assigning an impenetrable spherical envelope of radius 2.1 lattice units $(1.45 \AA)$ to each of the chain atoms representing a methylene group. Each head group was represented by a sphere of radius 2.4 lattice units. Intrachain effects were simulated by rejecting any chain conformations exhibiting overlap of spheres representing atoms separated by more than three bonds in the chain. The interchain excluded volume effects were implemented by occupying all the lattice sites within the sphere representing the atom.

The conformational dynamics of the lipid chain is considered to arise from a superposition of local structural rearrangements constrained by the bond lengths. The elemental move involves the transfer of a pair of adjacent atoms, $-\mathrm{C}_{1}-\mathrm{C}_{2}$ - chosen at random to different lattice sites. However, the motions of the head group atoms were penalized by a factor of 0.2 in order to account for their greater mass and to mimic the effects of their interaction with the aqueous phase. Each random elemental move attempted by the atoms is subjected to two acceptance tests:

(1) a move is only accepted if the final lattice positions of the excluded volume envelopes of the atoms are unoccupied;

(2) the new configuration was accepted with a probability $P$ given by the symmetric scheme

$$
P=\frac{\exp \left\{-E_{\text {new }} / k T\right\}}{\exp \left\{-E_{\text {new }} / k T\right\}+\exp \left\{-E_{\text {old }} / k T\right\}} .
$$

We note that the effects of temperature enter the simulation only through this acceptance test.

The Monte Carlo dynamics algorithm for a system containing $M$ hydrocarbon chains each consisting of $N$ atoms was executed in the following way. Given a particular configuration of the monolayer, $\Omega_{j}, M \times N$ local conformational moves are attempted at random with each atom in the system having an equal chance of being picked. These moves generate a new configuration of the monolayer, $\Omega_{j+1}$. The cycle is now repeated using $\Omega_{j+1}$ as the starting configuration. The fundamental time step of the algorithm is defined as the time required for the monolayer to undergo a transition from configuration $\Omega_{j}$ to $\Omega_{j+1}$. While the time step of the algorithm cannot be related to an absolute scale, a comparison with Brownian dynamics simulations strongly indicates that it maps linearly to real time [14].

We have typically generated a trajectory consisting of $16 \times 10^{3}$ monolayer configurations, each separated by 50 el- emental time steps at a temperature of $305 \mathrm{~K}$. A typical simulation run required between 16 and $28 \mathrm{~h}$ cpu time on a Dec Alpha 500au Personal Workstation.

\section{B. Coarse-grained continuum model}

This approach utilizes a coarse-grained representation of lipid molecules. Each chain is modeled as a flexible necklace of 5 beads $4 \AA$ in diameter, each corresponding to 3-4 successive methylene groups [21,22]. Effective intramolecular potentials are introduced to take into account chain connectivity and chain stiffness. However, the coarse-grained representation of the chain precludes the use of torsional potentials. The internal energy of the lipids contains two contributions as described previously [23,24]:

$$
U=U_{\text {bond }}+U_{\text {angle }} .
$$

The vibrational energy of the bonds along the chain backbone is

$$
U_{\text {bond }}=\sum_{\text {bonds }} \epsilon_{b}\left(b / b_{0}-1\right)^{2},
$$

where $\epsilon_{b}=250 \mathrm{kcal} / \mathrm{mol}$ and $b_{0}=3.9 \AA$. The bond length $b$ is only allowed to vary in the range $3.6 \AA \leqslant b \leqslant 4.2 \AA$. The bending energy of neighboring bonds was taken to be of the form

$$
U_{\text {angle }}=\sum_{\text {angles }} \epsilon_{\theta}(1+\cos \theta)^{2}
$$

where $\theta$ is the instantaneous valence angle and $\epsilon_{\theta}$ $=15 \mathrm{kcal} / \mathrm{mol}$.

The monolayer contained 1000 model lipid chains in a simulation cell with periodic boundary conditions in the $x-y$ planes and extending along the positive $z$ axis. The first bead of each chain was tethered to an impenetrable surface at $z$ $=0$. This was realized by subjecting the bead to a potential $U(z)$ of the form

$$
U(z)=\left\{\begin{array}{rr}
0 & \text { for } 0<z<\sigma_{L} \\
\infty & \text { for } z>\sigma_{L}
\end{array}\right.
$$

Here $\sigma_{L}$ is the diameter of the bead ( $4 \AA$ ) . Thus, the lipid heads are bound but can diffuse in close proximity to the interface. Excluded volume effects were mimicked by the steric potential

$$
U_{\text {steric }}=\sum_{i, j=1} V\left(r_{i j}\right)
$$

where $V$ is a hard-sphere potential

$$
V\left(r_{i j}\right)= \begin{cases}0 & \text { for }\left|\mathbf{r}_{i}-\mathbf{r}_{j}\right|>\sigma, \\ \infty & \text { for }\left|\mathbf{r}_{i}-\mathbf{r}_{j}\right|<\sigma,\end{cases}
$$

with $\sigma=\sigma_{L}$. This potential was used for intermolecular interactions and for interactions between monomers separated by more than one bond in the same molecule.

The numerical implementation of the algorithm follows a metropolis scheme. A lipid molecule is randomly selected at 
each trial and it is attempted to move it from its original position by randomly displacing a selected bead from the chain. Each move is accepted if

$$
\exp (-\Delta U / R T)>\eta
$$

where $0<\eta<1$ is a random number and $\Delta U$ is the difference between the old and the new energies of the system. The simulations were carried out at a temperature of $T$ $=305 \mathrm{~K}$. One Monte Carlo step (MCS) corresponds to 5000 trials and defines the time scale. The maximum size of the trial move was adjusted so as to obtain an acceptance rate of at least 50\%. A link-cell method [25] was implemented in the algorithm for the efficient sampling of $\Delta U$. The initial random configuration of the lipids is equilibrated at constant volume for $2 \times 10^{5} \mathrm{MCS}$.

\section{RESULTS AND DISCUSSION}

The long-time behavior of a lipid monolayer containing 1000 chains was simulated using two different approaches: the knight's gambit moves (KGM) model and a coarsegrained continuum (CGC) model. The simulations were carried out as a function of the area per molecule of the model lipid chains. For the CGC model, the area per molecule varied between 17 and $1350 \AA^{2}$, while for the KGM simulations, the range was 17-1200 $\AA^{2}$. The lowest area per molecule used corresponded to a monolayer configuration just above the order-disorder transition of the chains. We note here that the temperatures used in the two models are not directly comparable, since the two models use different representations of the chains and their conformational potentials.

\section{A. Conformational disorder of the lipid chains}

The disordered conformational states of the model lipid chains is conveniently monitored by the change in the height distribution of chain atoms (beads) above the monolayer interface, shown in Figs. 1(a) and 1(b) for the KGM and CGC models, respectively. It can be seen that the central and the terminal beads can access a broad distribution of heights above the monolayer interface at $z=0$. The distributions broaden significantly, though to different extents, on increasing the area per molecule of the model chains. The simulations show further that the radii of gyration of the chains change significantly over the range of areas per molecule studied.

We stress here that the detailed conformational behavior undertaken by the model chains is treated in different ways in the two models and that this is reflected not only in the height distributions but also in the orientational order parameters. In view of the coarse-grain representation used in the CGC model, we shall only consider the order parameters of the bonds joining successive atoms (beads) of the model chains, Figs. 2(a) and 2(b) for the KGM and CGC models, respectively. The order parameter $S(n)$ is defined as
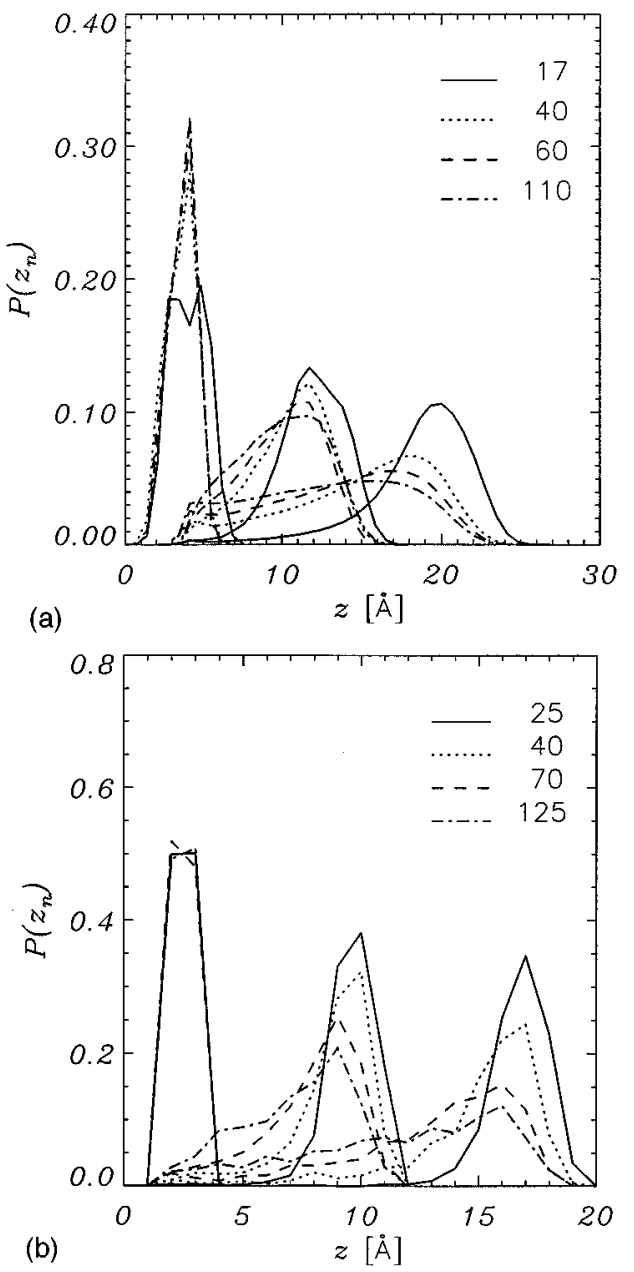

FIG. 1. (a) Distribution heights above the monolayer surface of different atoms along the model chain of the KGM model at different areas per molecule. Left, head group; middle, atom 9; right, terminal atom 16. (b) Height distribution of the head group (left), bead 3 (middle), and terminal bead 5 (right) for the CGC model.

$$
S(n)=\frac{1}{2}\left\langle 3\left[\left(z_{n+1}-z_{n}\right) / a_{n}\right]^{2}-1\right\rangle,
$$

where $a_{n}$ is the length of the bond joining atoms (beads) $n$ and $n+1$ and $z_{n}$ its $z$ component. It can be seen from Fig. 2 that the bond order parameter decreases more rapidly along the KGM chains than the CGC ones. The orientational disorder revealed by $S(n)$ is determined by the packing of the chains in the monolayer as well as by the torsional potential used in the KGM model and the bending potential in the CGC model. The question now arises as to whether the potential or the packing determines the chain configurations. To this end, we simulated the bond order parameter profile of an athermal chain $(T \rightarrow \infty)$. The profiles of the bond order parameters at different areas per molecule for the two models, Figs. 3, are found to exhibit the same trends as those shown in Fig. 2 for the chains at $T=305 \mathrm{~K}$. In general, the bond order parameters of the athermal chain are lower than that of the chain at $305 \mathrm{~K}$ for the same area per molecule. However, the simulations show that the profiles of the latter chains can be recovered on compressing the monolayer of the athermal 


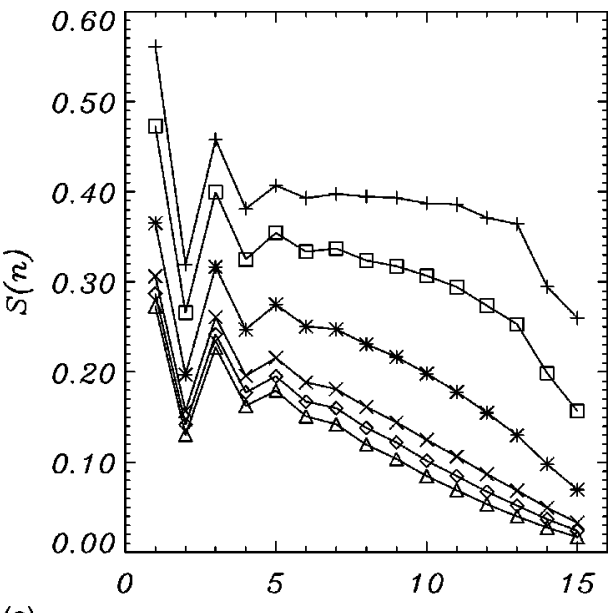

(a)

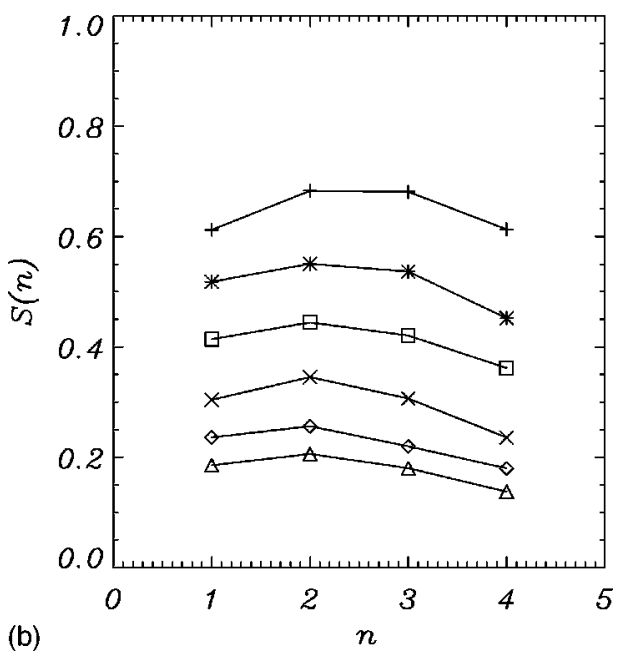

FIG. 2. (a) Variation of the order parameter $S(n)$ [Eq. (11)] of the bond vectors along the chains of the KGM model at different areas per molecule $a:+=17 \AA^{2} ; \square, 24 \AA^{2} ; *, 39 \AA^{2} ; \times, 58 \AA^{2}$; $\diamond, 69 \AA^{2} ; \triangle, 80 \AA^{2}$. (b) as in (a) but for chains of the CGC model $a:+, 30 \AA^{2} ; \quad *, 40 \AA^{2} ; \quad \square, 50 \AA^{2} ; \quad \times, 70 \AA^{2} ; \quad \diamond, 90 \AA^{2} ;$ $\triangle, 110 \AA^{2}$.

chains. These findings indicate that the packing of the chains essentially determines their disordered conformations.

\section{B. Lateral motion over the monolayer surface}

It should now be noted that the lateral diffusion of the model lipid chains in our simulations arises solely as the result of the superposition of local intramolecular conformational transitions. The local conformational changes implemented in the simulations are considerably faster than the overall motions of the chains over the monolayer interface. As a result of this effective time-scale separation, we may view the lateral diffusion process as the stochastic motions of the model chains in a time-averaged conformation. Moreover, the results shown in Figs. 1 and 2 indicate that this average conformation changes significantly on increasing the effective area per molecule in the monolayer.

In view of the disordered conformational state of the lipid chains, we characterized their lateral motion in the $x-y$ plane by following the positions of the head groups and centers of mass (CM's) during the simulation. These trajectories of the

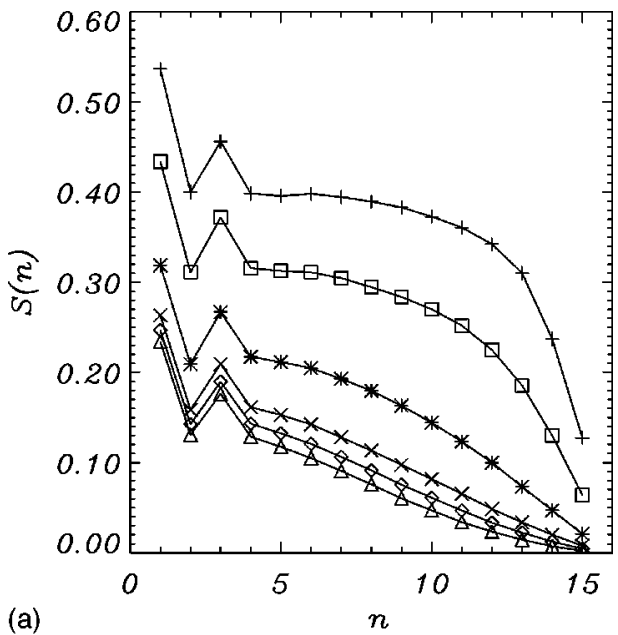

(a)

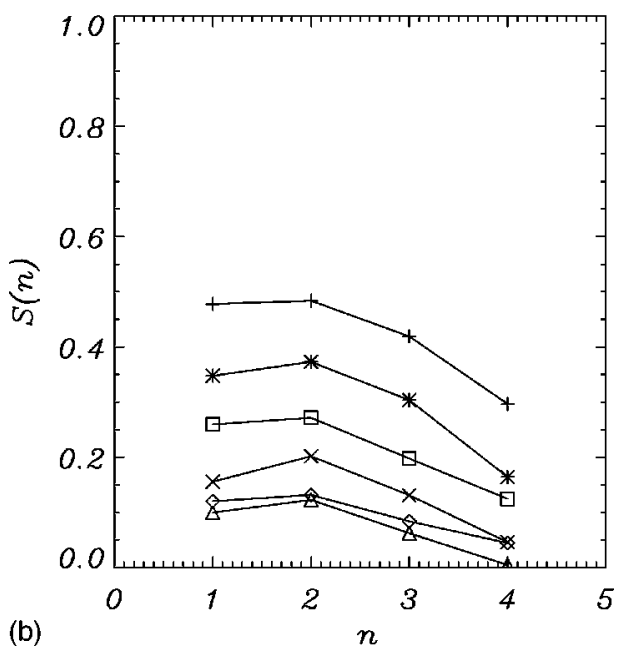

FIG. 3. Same as Fig. 2 for athermal chains.

displacements show clearly that the movement of the head group can be superimposed on that of the CM. The linear relation found between the positions of the head group and $\mathrm{CM}$ along the trajectory is shown in Fig. 4 for the KGM model. We shall therefore only consider the lateral motion of the head groups in the following discussion.

The mean square displacement of the head groups from their positions at the start of the simulation, $s(t)=\langle(r(t)$ $\left.-r(0))^{2}\right\rangle$, was monitored during the simulation. Note that

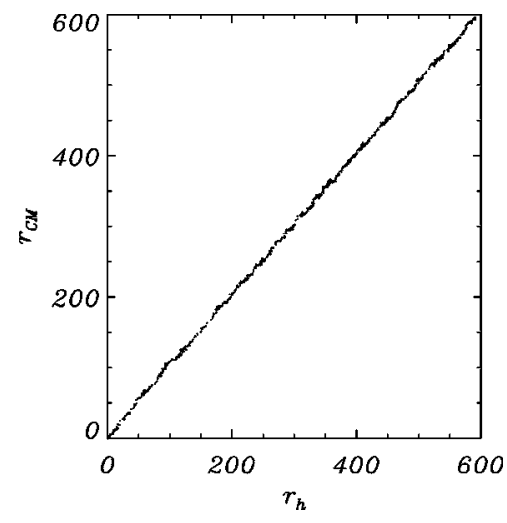

FIG. 4. Correlation between the displacement of the head group, $r_{h}$, of a single KGM chain and the displacement of its center of mass, $r_{C M}$, during a simulation run. 


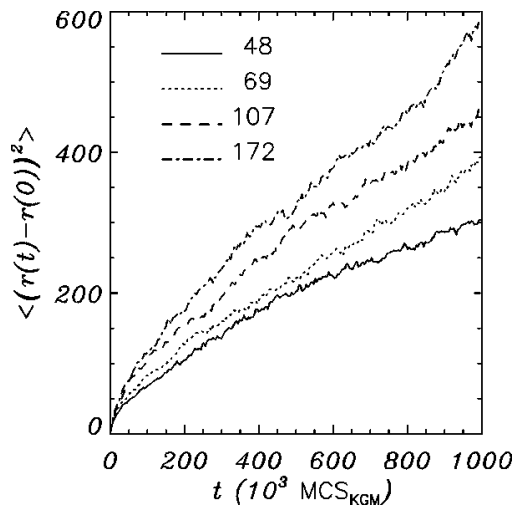

FIG. 5. Initial time evolution of the mean square displacement of the head groups of the chains of the KGM model at different areas per molecule.

$s(t)$ was averaged over different starting times. At short times, where the displacements are of the order of the area per molecule, a parabolic time increase in $s(t)$ is often observed; see Fig. 5. This is indeed expected, since the internal conformational dynamics will influence the lateral motions in this time regime. The Einstein behavior, with $s(t)$ increasing linearly with time, is recovered when the displacements become comparable to the molecular cross section, Fig. 6. The simulations thus indicate that the effective diffusion coefficient at short times is larger than that determined at long times. Such behavior has indeed been observed experimentally $[8,10]$.
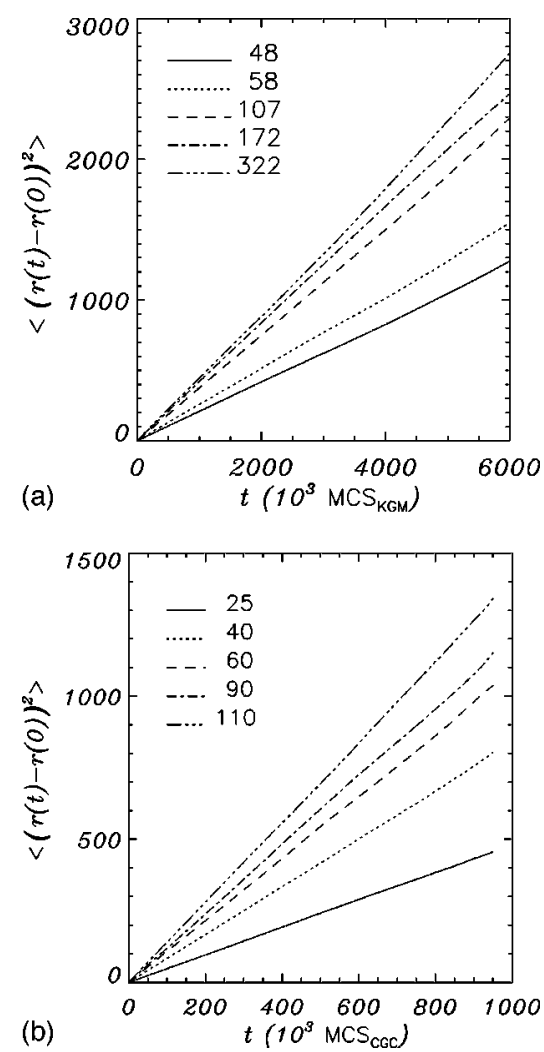

FIG. 6. Time evolution of the mean square displacement at longer times, where the Einstein relation is recovered (a) for the KGM model; (b) for the CGC model. Note that the Monte Carlo time scales of the two models are not related.

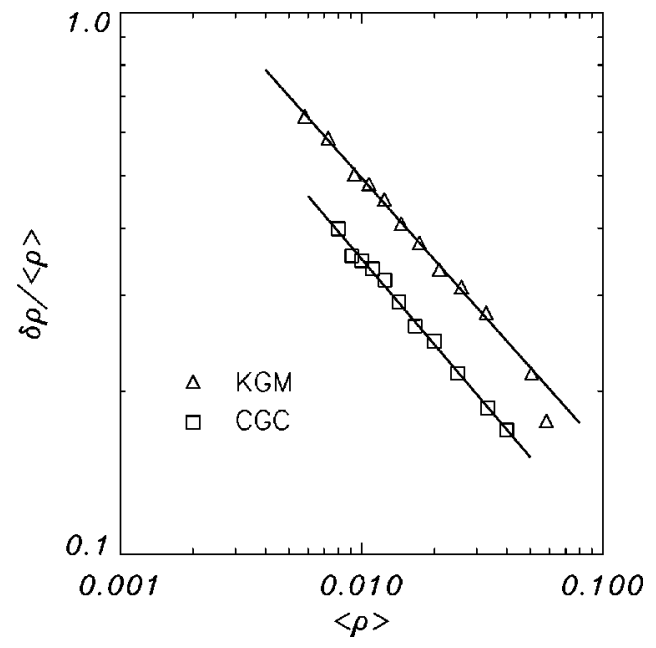

FIG. 7. Variation of the normalized number density fluctuations in the monolayer, [Eq. (12)], with the inverse area per molecule for the KGM and CGC models. The continuous lines show the powerlaw fits to the data.

The lower limit of the area per molecule used in the simulations was dictated by the observation that when the model chains are close-packed, the mean square displacements reach a plateau at quite short times. This behavior indicates that the lateral motions of the chains are confined to small local domains within the monolayer. We have not considered these cases further. Nevertheless, we note that the behavior observed here is in excellent agreement with the findings of Saxton [26-28], for the case of two-dimensional (2D) diffusion of hard objects.

The simulations show that the time evolution of the lateral displacements of the lipid molecules is sensitive to the details of the model for conformational motions used in the simulation. This has indeed been reported previously in molecular dynamics studies of liquid alkanes [29]. The KGM and CGC models thus yield different magnitudes for the lateral diffusion coefficients. Moreover, the Monte Carlo time scales cannot be related in an obvious way. The success of

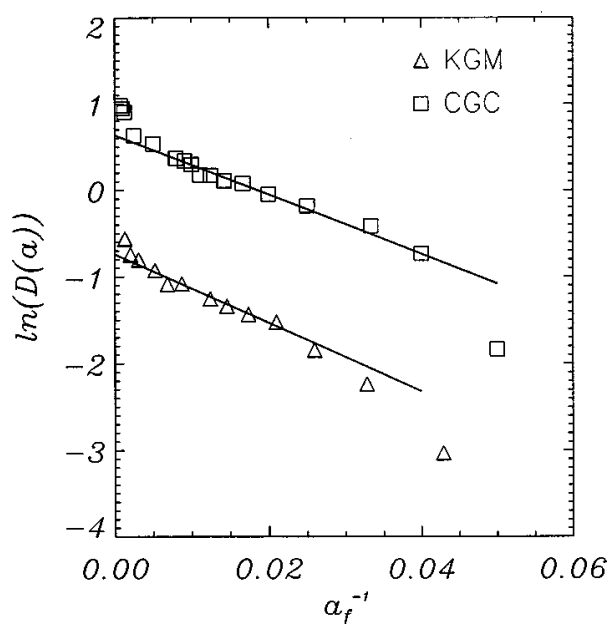

FIG. 8. Dependence of the lateral diffusion coefficients on the area per molecule obtained from simulations with the KGM and CGC models by using the linearized form of theoretical relation derived for a two-dimensional fluid of hard disks. 
the models, however, can be gauged by the agreement of their predictions with experimental observations, such as those reported by Peters and Beck [12]. In doing this we are conscious of two possible shortcomings in the simulations. In the first place, the size of the system is small compared to that employed experimentally. Second, the neglect of intermolecular and hydrodynamic interactions in the simulation may affect our findings.

Further insight into the process of lateral diffusion of the lipid molecules can be obtained from the normalized number surface density fluctuations [30],

$$
\delta \rho /\langle\rho\rangle=\sqrt{\left\langle\rho^{2}\right\rangle-\langle\rho\rangle^{2}} /\langle\rho\rangle
$$

where $\langle\rho\rangle=1 / a$ and $a$ is the area per molecule. $\delta \rho /\langle\rho\rangle$ is evaluated on dividing the Monte Carlo simulation box into 25 identical cells and monitoring the fluctuations in the number of head groups in each one. As shown in Fig. 7, $\delta \rho /\langle\rho\rangle$ exhibits the power-law dependence on $\langle\rho\rangle$ expected from thermodynamic considerations over the entire range used. The values of the exponent, -0.53 for the CGC model and -0.51 from the KGM model, compare favorably with the expected value [30] of $-1 / 2$. It thus turns out that both models yield density fluctuations that are essentially independent of the packing and conformational states of the model lipid molecules in the monolayer.

\section{Variation of lateral diffusion coefficients with area per molecule}

\section{Analysis in terms of a $2 D$ hard-disk system}

The lateral diffusion coefficients $D(a)$ of the model lipid chains were obtained from $s(t)$ as a function of the area per molecule $a$. As with experimental studies of lateral diffusion [12], we shall here analyze the dependence of $D(a)$ on $a$ using the analytical relation derived for a two-dimensional hard-disk fluid. Here the diffusion constant is related to the free area $a_{f}$ as $[11,13]$

$$
D(a)=D\left(r^{*}\right) \exp \left(-\gamma a^{*} / a_{f}\right),
$$

where $a_{f}=a-a_{H C}$ and $a_{H C}=\pi r^{* 2}$ ( $a_{H C}$ is the hard-core area of the diffusing molecule), $r^{*}$ is its hard-core radius, and $\gamma$ is a constant accounting for the overlap of free volume (or free area). $a^{*}$ is the critical free area at which the displacement becomes possible. The pre-exponential factor $D\left(r^{*}\right)$, the diffusion coefficient of a single disk, depends, in general, on temperature and viscosity. This model rests on the assumption that a hopping process underlies the lateral diffusion of the chains. Three steps are involved: (1) creation of a hole in the structure by lateral density fluctuations; (2) a molecular jump into this hole; (3) filling of the resulting void.

The main conceptual difficulty in applying the results of the two-dimensional hard-disk theory to the results of our three-dimensional simulation is the formulation of an appropriate definition for the hard-core area of the diffusing species. We have shown above that the model lipid chains are conformationally disordered and, moreover, that the timeaveraged radius of gyration changes with the area per molecule. The simulations thus indicate that the effective hard- core area of the diffusion chains depends on the area per molecule, in contradiction with the theoretical assumptions. The lateral diffusion of the model chains arises in our simulations solely as the result of local conformational transitions. These moves can only be executed if free volume cavities exist at the destination position. The cavities, in turn, are created and destroyed by density fluctuations arising from the conformational disorder of the chains. We have shown above that the simulations provide substantive evidence for the view that the density fluctuations in the monolayer are independent of the area per molecule.

The simulated diffusion coefficients $D(a)$ were fitted numerically to the linearized form of the theoretical relation on taking $a_{H C}$ as an adjustable parameter [12]:

$$
\ln [D(a)]=\ln \left[D\left(r^{*}\right)\right]-\gamma^{\prime} /\left(a-a_{H C}\right) .
$$

Interestingly, the best fits over the entire range of $a_{f}$ were obtained with $a_{H C}=0$, Fig. 8, albeit with significant systematic deviations. Nevertheless, the fits yield an acceptable description of the diffusion coefficients, particularly at moderate densities of the chains in the monolayer. This finding demonstrates the inadequacies of the simple twodimensional theories in describing the physical processes underlying the lateral motion of flexible molecules over a planar surface.

The type of behavior found in the simulations closely parallels the experimental observations of Peters and Beck [12]. In particular, the analytical theory systematically underestimates the lateral diffusion coefficients at high areas per molecule (low densities). There have been a number of attempts to clarify the origin of the observed deviations. A particularly appealing explanation, put forward by Nonnenmacher [13], attributes the discrepancies to the fact that the theoretical relationship was derived on the assumption that the density fluctuations responsible for the hole creation are characterized by a single amplitude. The introduction of density fluctuations with a broad range of amplitudes was shown to provide a quantitative description of the experimental data over the entire range of areas per molecule.

The deviations from the simple exponential behavior found in the simulations may be understood in terms of the Nonnenmacher model [13]. It is clear that at low areas per molecule, large amplitude density fluctuations are needed to create the free volume cavities needed for the lateral diffusion process. The free volume cavities increase in size and are more easily formed as the area per molecule increases. Now, the amplitude of the density fluctuations needed to bring about the hole creation decreases. This causes an enhancement in the lateral diffusion rate, since small amplitude fluctuations are expected to occur more frequently than large amplitude ones.

\section{CONCLUSIONS}

Lateral diffusion of conformationally disordered lipid chains over the surface of a monolayer was simulated using dynamic Monte Carlo techniques. Two models employing different representations of the internal motions of lipid chains were used. The results indicate that the dependence of the lateral diffusion coefficients on the area per molecule in 
the monolayer is determined by the conformational behavior of the lipid chains. The two-dimensional model commonly used for analysis of the lateral diffusion process was found to provide an inadequate description of the simulated data. The results presented here are in line with the fractal description of the diffusion process introduced by Nonnemancher and underscore the three-dimensional nature of the diffusion process.

\section{ACKNOWLEDGMENT}

Financial support to T.S. from DGICYT (Spain) under Project No. PB94-1167 is gratefully acknowledged.
[1] M. Edidin, in The Structure of Biological Membranes, edited by P. Yeagle (CRC Press, Boca Raton, FL, 1992), pp. 539572.

[2] F.G. Zhang, G.M. Lee, and K. Jacobson, BioEssays 15, 579 (1993).

[3] D.F. Kucik, E.L. Elson, and M.P. Sheetz, J. Cell Biol. 111, 1617 (1990).

[4] R. Peters, FEBS Lett. 234, 1 (1988).

[5] J. Lavergne and P. Joliot, Trends Biochem. Sci. 16, 129 (1991).

[6] J. Lavergne, J.P. Bouchaud, and P. Joliot, Biochim. Biophys. Acta 1101, 13 (1992).

[7] K. Jacobson and W.L.C. Vaz, Comments Mol. Cell. Biophys. 8, 1 (1992).

[8] P.F.F. Almeida and W.L.C. Vaz, in Structure and Dynamics of Membranes. From Cells to Vesicles, edited by R. Lipowsky and E. Sackmann (Elsevier, Amsterdam, 1995), pp. 305-357.

[9] L. Rilfors, P.-O. Eriksson, G. Arvidsen, and G. Lindblom, Biochemistry 25, 7702 (1986).

[10] C. Dolainsky, P. Karakatsanis, and T.M. Bayerl, Phys. Rev. E 55, 4512 (1997).

[11] M.H. Cohen and D. Turnbull, J. Chem. Phys. 31, 1164 (1959).

[12] R. Peters and K. Beck, Proc. Natl. Acad. Sci. USA 80, 7183 (1983).

[13] T.F. Nonnenmacher, Eur. Biophys. J. 16, 375 (1989).

[14] A. Rey, A. Kolinski, J. Skolnick, and Y.K. Levine, J. Chem. Phys. 97, 1240 (1992).
[15] Y.K. Levine, A. Kolinski, and J. Skolnick, J. Chem. Phys. 98, 7581 (1993).

[16] U.A. Van der Heide and Y.K. Levine, Biochim. Biophys. Acta 1195, 1 (1994).

[17] D.A. Van der Sijs and Y.K. Levine, J. Chem. Phys. 100, 6783 (1994).

[18] H. Eviatar, U.A. Van der Heide, and Y.K. Levine, J. Chem. Phys. 102, 3135 (1995).

[19] M.A.M.J. Van Zandvoort, H.C. Gerritsen, and Y.K. Levine, J. Phys. Chem. B 101, 4142 (1997)

[20] J.P. Ryckaert and A. Bellemans, Chem. Phys. Lett. 30, 123 (1975).

[21] F.M. Haas, R. Hilfer, and K. Binder J. Chem. Phys. 102, 2960 (1995).

[22] T. Sintes and A. Baumgärtner, Biophys. J. 73, 2251 (1997).

[23] T-X. Xiang, Biophys. J. 65, 1108 (1993).

[24] A. Baumgärtner, J. Chem. Phys. 103, 10669 (1995).

[25] M.P. Allen and D. Tildesley, Computer Simulation of Liquids (Clarendon, Oxford, 1987).

[26] M.J. Saxton, Biophys. J. 64, 1766 (1993).

[27] M.J. Saxton, Biophys. J. 66, 394 (1994).

[28] M.J. Saxton, Biophys. J. 70, 1250 (1996).

[29] M. Mondello, G.S. Grest, E.B. Weber III, and P. Peczak, J. Chem. Phys. 109, 798 (1998).

[30] J.P. Hansen and I.R. McDonald, Theory of Simple Liquids (Academic Press, London, 1986). 